\title{
Investigation on Temporal Skewing among O-CDMA Code Carriers under Fiber Temperature Variations
}

\author{
Mohamed Abuhelala, Md Shakil Ahmad, Mustafa Ibrahim and Ivan Glesk, Senior Member, IEEE
}

\begin{abstract}
Experimental investigation has been conducted on the influence of optical fiber temperature variations on Two Dimension Wavelength/Hopping Time/Spreading Incoherent Optical Code Division Multiple Access (2D-WH/TS iOCDMA) codes. Our experimental results show that in the literature reported approaches assuming a temperature induced coefficient $D_{\text {temp }}$ in optical fiber being a constant are only accurate when the selection of code carriers' wavelengths is from a relatively narrow spectral interval. This paper shows that if the selection of code carriers' wavelengths is from a wider spectral interval, neglecting to mitigate the wavelength dependency of $D_{\text {temp }}$ would have a significant impact on the recovered iOCDMA Autocorrelation function.
\end{abstract}

Keywords - Dispersion temperature coefficient, incoherent OCDMA autocorrelation function, spectral spacing, time skewing.

\section{INTRODUCTION}

$\mathrm{T}$ WO Dimension Wavelength/Hopping Time/Spreading Incoherent Optical Code Division Multiple Access (2D-WH/TS iOCDMA) based on picosecond code carriers has one important challenge - a code carriers time skewing. This effect is the result of relative group velocity dispersions delays caused by the different wavelength used by the code [1]. Group velocity dispersion is also the cause of pulse broadening and the recovered autocorrelation function (peak) power reduction at the receiving side [2]. The loss of peak power can be overcome by amplifying [3]. In attempting to reduce the effect of broadening, ultranarrow carriers' linewidth can be exploited as a possible solution. An investigation by simulations has been done on time skewing effects among narrow pulses of $0.1 \mathrm{~nm}$ in $2 \mathrm{D}$ WH/TS iOCDMA system. However, adding time skewing effect to that evaluation showed significant reduction of the system maximum transmission distance of $15 \mathrm{~km}$ [4].

This project has received funding from the European Union's Horizon 2020 research and innovation program under the Marie Skłodowska-Curie grant agreement No 734331.

Mohamed S. Kh. Abuhelala is with the Department of Electronic and Electrical Engineering, University of Strathclyde, 204 George Street, Glasgow G1 1XU, United Kingdom (phone: +44 7721534491; e-mail: mohamed.abuhelala@strath.ac.u).

Md Shakil Ahmad is with the Department of Electronic and Electrical Engineering, University of Strathclyde, 204 George Street, Glasgow G1 1XU, United Kingdom (phone +445482528; e-mail shakil.ahmed@ strath.ac.uk)

Mustafa Ibrahim is with the School of Electrical and Electronic Engineering, Glasgow Caledonian University, Glasgow, United Kingdom (e-mail: mustafa.ibrahim@caledonian.ac.uk).

Ivan Glesk is with the Department of Electronic and Electrical Engineering, University of Strathclyde, Glasgow G1 1XW, United Kingdom (+441415482529; e-mail: ivan.glesk@strath.ac.uk).
Various studies have shown the importance of understanding the effects of fiber temperature fluctuations induced by the fiber surrounding environment. The temperature induced dispersion variations can be neglected for system operates in low data rates. Considering upgrading current systems to a higher speed data rate, for example to $40 \mathrm{~Gb} / \mathrm{s}$, the temperature induced dispersion variation must not be ignored [5]-[7]. However, in a manufacturing process, production is typically conducted at the room temperature. Therefore, it is essential to understand the relationship between a temperature and dispersion and to also study the performance of fiber optic systems operating in the environment which is significantly different from that during the manufacturing [8].

Several techniques have been proposed to mitigate the impact of the temperature induced dispersion and used to mitigate a relatively small amount of skewing [3], [9]. However, when considering using a wider spectral range for communication applications, the amount of skewing will be significantly larger.

To the best of our knowledge, in this paper, we report for the first time an investigation on the wavelength dependency of the temperature induced fiber dispersion coefficient $D_{\text {temp }}$ in cases when short picosecond code carriers are selected from a much wider spectral interval.

\section{THEORETICAL BACKGROUND}

To understand the effect of temperature induced fluctuations on the optical pulse position during the transmission, Osadola [6] has studied temporal skewing $\Delta t$ of code carriers (see Eq. (1)) in 2D-WH/TS iOCDMA system. From the experimental study [10], which agrees with our results for SMF-28 fiber, $\Delta t$ decreases with the increase of the fiber temperature. Equation (1) holds for cases when

$$
\Delta t=D_{\text {temp }} \times L \times \Delta \Lambda \times \Delta \mathrm{T}
$$

the spectral interval for selecting code wavelength carriers is considered to be small (only a few $\mathrm{nm}$ ).

In order to be more general, (1) needs to be modified by the following way:

$$
\Delta t_{n}=D\left(\lambda_{\mathrm{n}}\right)_{\text {temp }} \times L \times \Delta \Lambda \times \Delta \mathrm{T}
$$

where, $\mathrm{n}=1,2,3, \ldots, w$. Here, $w$ is the code weight [6]. $D\left(\lambda_{\mathrm{n}}\right)_{\text {temp }}$ is the temperature induced dispersion coefficient in ps $/ \mathrm{nm} \cdot \mathrm{km} \cdot{ }^{\circ} \mathrm{C}, L$ is the fiber length in $\mathrm{km} . \Delta \Lambda$ is the spectral spacing between adjacent wavelengths code carriers defined as

$$
\Delta \Lambda=\lambda_{\text {ref }}-\lambda_{\mathrm{n}}
$$

where $\lambda_{\text {ref }}$ is the longest wavelength and will be called a 
reference wavelength. $\Delta T$ is a fiber temperature change and is defined as

$$
\Delta T=T_{\text {initial }}-T_{\text {final }}
$$

For calculating $D\left(\lambda_{\mathrm{n}}\right)_{\text {temp }}$ from Eq. (2), we have used the obtained experimental results.

The recovered auto-correlation function for 2D-WH/TS iOCDMA affected by the skewing and dispersion due to the fiber temperature changes has been found as [6]

$$
S_{t}(L)=\sum_{k=0}^{w-1} P_{p} e^{\left\{-2.77\left[\frac{t-(k \Delta t)}{\tau-\Delta \tau}\right]^{2}\right\}}
$$

where the constant -2.77 reflects its FWHM values and $w$ is a number of wavelength code carriers (also known as a codes weight). $P_{p}$ is the transmitter power; $t$ is the 2DWH/TS iOCDMA code-word length (its duration) in picoseconds, ps, and $\Delta t$ is the amount of the pulse width change also in picoseconds. For the illustration, see Fig. 1.

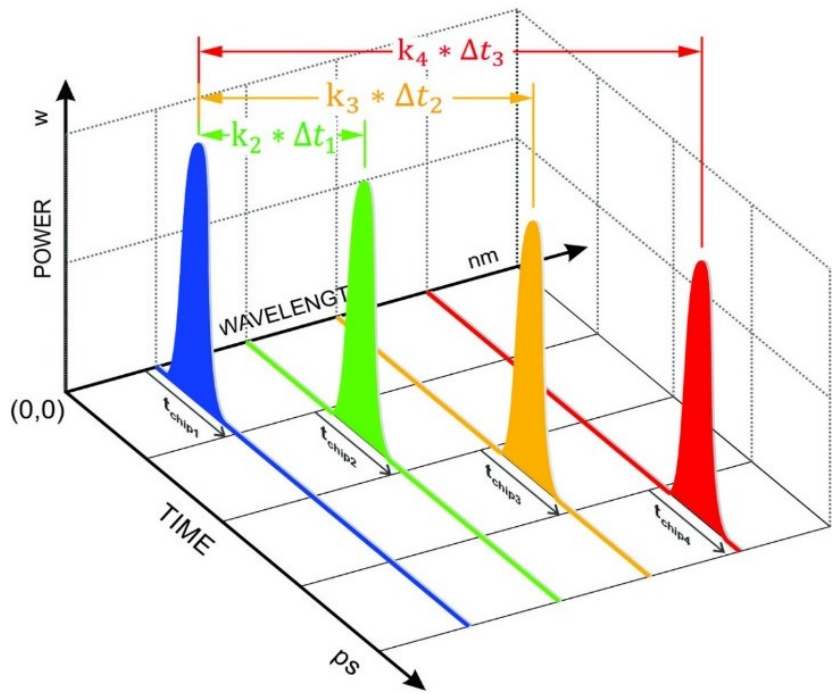

Fig. 1. Illustration of the 2D-WH/TS iOCDMA code.

\section{SYSTEM SETUP}

As it is shown in Fig. 1, a Mode-Locked Laser (MLL) generates optical pulses with a central wavelength of 1545 $\mathrm{nm}$ having a linewidth of $2.6 \mathrm{~nm}$. Supercontinuum Generator (SCL) is then used to produce an optical supercontinuum with its central wavelength of $1550 \mathrm{~nm}$ and the linewidth of $3.2 \mathrm{~nm}$. By using a Fiber Bragg Grating (FBG) OCDMA encoder (OKI Industries, Japan) four optical pules are produced (carved from the supercontinuum) $\lambda_{1}=1550.92 \mathrm{~nm}, \quad \lambda_{2}=1550.12 \mathrm{~nm}, \quad \lambda_{3}=$ $1551.72 \mathrm{~nm}$, and $\lambda_{4}=1552.52 \mathrm{~nm}$. Each wavelength pulse has a $0.8 \mathrm{~nm}$ linewidth. The optical pulse at $1545 \mathrm{~nm}$ is then delayed in time by an optical delay line and injected along with all four wavelengths $\lambda_{1}$ to $\lambda_{4}$ by using a $2 \times 1$ optical fiber coupler. This arrangement would allow us to study the individual relative position changes $\Delta t$ of the pulses while being propagated in an optical fiber spool made of $19.5 \mathrm{~km}$ long Standard Single Mode Fiber (SMF-28) made by Corning, Inc. The spool is housed in an environmental chamber (EC) SM-32C from Thermatron Industries (see Fig. 2 to introduce the desired temperature changes to SMF28. The fiber link is fully compensated for chromatic dispersion by using a commercial Dispersion Compensated Fiber (DCF). The temporal skewing due to the influence of fiber temperature changes was observed using an Agilent Oscilloscope (86100C) with an optical sampling head of a limited bandwidth of $65 \mathrm{GHz}$.

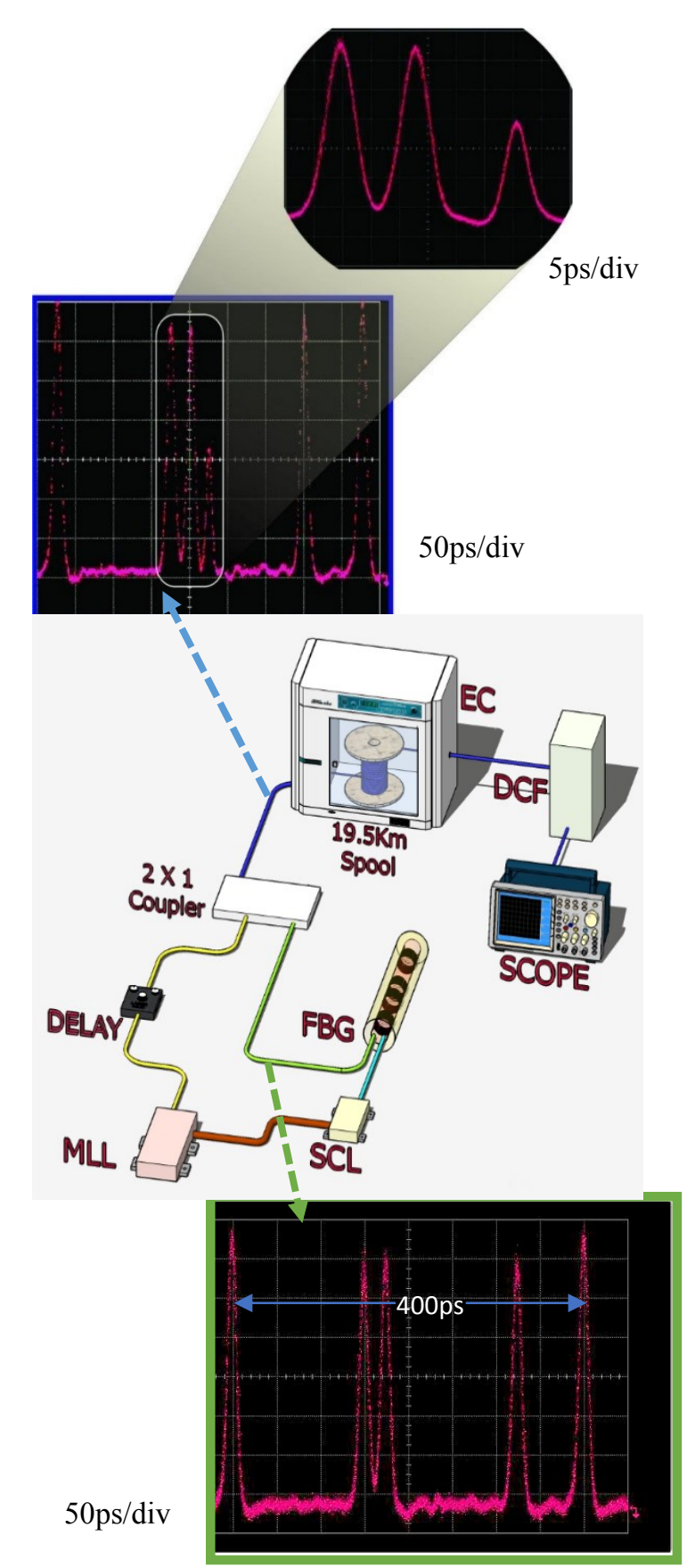

Fig. 2. Experiment setup. EC -Environmental Chamber, DCF - Dispersion Compensating Fibre, Scope Agilent Oscilloscope 86100C, FBG - Fibre Brag Grating, SCL - Supercontinuum source, MLL - Erbium doped fiber mode locked laser.

\section{OBTAINED EXPERIMENTAL RESULTS}

The main goal of this investigation was to examine the accuracy of the Eq. (1 )and then determine if using constant (i.e., wavelength independent) value of $D_{\text {temp }}$ for the temperature induced dispersion coefficient in optical fiber, would be suitable also for a broader range of wavelengths if they are used as the optical code carriers in 2D-WH/TS iOCDMA system. The approach we followed in this work was using three wavelengths $\left(\lambda_{1}=1540 \mathrm{~nm}, \lambda_{2}=1550.12 \mathrm{~nm}\right.$, $\left.\lambda_{\text {ref }}=1551.72 \mathrm{~nm}\right)$. Wavelength $\lambda_{\text {ref }}$ was used as the reference for calculating relative delays $\Delta t$ between the individual wavelength pulses' positions. 
Initially, the delay was fixed, for $\Delta t_{1}=27 \mathrm{ps}$ and for $\Delta t_{2}=36 \mathrm{ps}$, respectively. The fiber temperature was $T=0^{\circ} \mathrm{C}$. This can be seen in Fig. 3 .

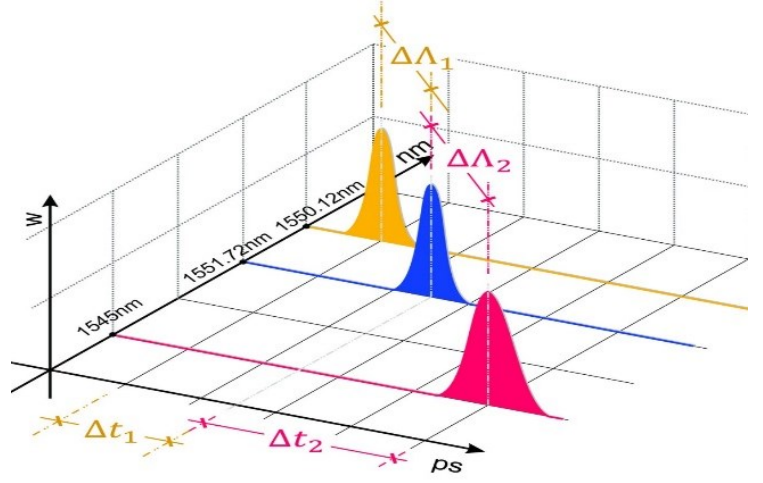

Fig. 3. Illustrates the concept of delays selection.

To investigate the limitations of Eq. (1), we used two different values for spectral spacing, a "narrow spectral spacing' of $1.6 \mathrm{~nm}$ in case of $\Delta t_{1}$ delay and a 'wide spectral spacing' of $6.72 \mathrm{~nm}$ for $\Delta t_{2}$ delay. In this investigation, the temperature induced coefficient of the dispersion compensated fibre $\left(D_{D C F}\right)$ was neglected because it has a very small impact in the calculation $D_{\mathrm{DCF}}=$ $0.0009 \mathrm{ps} / \mathrm{km} \bullet \mathrm{nm} \bullet{ }^{\circ} \mathrm{C}[5]$.

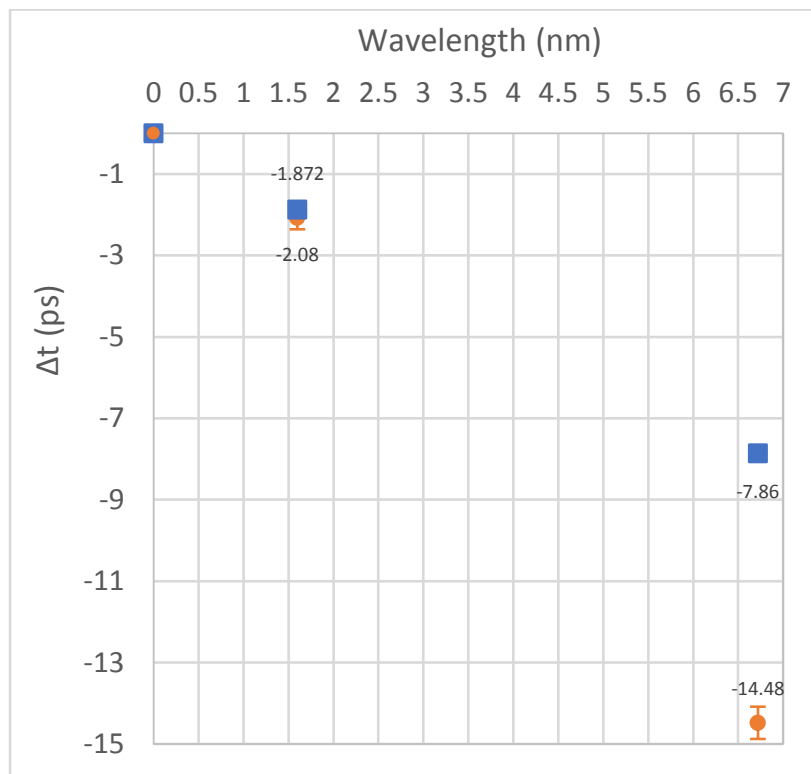

Fig. 4. Illustration of the impact of $\Delta t$; blue squares define $\Delta t_{1} ; \Delta t_{2}$ was calculated using Eq. (2). Red circles define $\Delta t_{1} ; \Delta t_{2}$ was obtained from our measurements. The standard deviation for $\Delta t_{1}$ is $0.277 \mathrm{ps}$ and for $\Delta t_{2}$ is $0.396 \mathrm{ps}$, respectively.

To ensure the experimental setup can support obtaining accurate results, we take in to account the following:

- In each measurement for obtaining $\Delta t_{1}$ and $\Delta t_{2}$ MLL and SCL settings were always rechecked by an optical spectral analyzer to insure the same linewidth is maintain across all measurements.

- Measurements were taken for temperatures from $0^{\circ} \mathrm{C}$ to $40^{\circ} \mathrm{C}$ with steps of $10^{\circ} \mathrm{C}$ in each data taking, the temperature in the Environmental Chamber was let to stabilize for three and half hours before changing the temperature settings.

- Each measurement was repeated 5 times for the same temperature. We used average values for obtaining $\Delta t_{1}$ and $\Delta t_{2}$.

\section{DISCUSSION}

It is important to realize that the chromatic dispersion is mostly depends on the fibre material [8]-[11]. Although, there are several types of SMF on the market, the temperature variation dependence of $D_{\text {temp }}$ for SMFs does not 'hugely' changes from fibre to fibre [5] and is known to be $-0.0015 \mathrm{ps} / \mathrm{nm} \cdot \mathrm{km} \bullet{ }^{\circ} \mathrm{C}[10]$. We have experimentally tested the equation by considering two spectral spacing, $1.6 \mathrm{~nm}$ and $6.72 \mathrm{~nm}$, respectively. When using the narrower spectral spacing of $1.6 \mathrm{~nm}$ our measurements for $\Delta t_{1}$ perfectly agree with the literature [3], [12]. However, for the large spectral spacing between wavelengths, the measurement data related to $\Delta t_{2}$ do not agree with the former. From Fig. 4 , the calculated $\Delta t_{2}$ if based on the temperature induced dispersion coefficient known from the literature would be $\Delta t_{1}=-7.86 \mathrm{ps}$. Whereas, based on our measurements, $\Delta t_{2}=-14.48 \mathrm{ps}$. This then leads to a temperature induced dispersion coefficient found based on Eq. (2) to be $D_{\text {temp }}=-0.0027 \mathrm{ps} / \mathrm{nm} \cdot \mathrm{km} \cdot{ }^{\circ} \mathrm{C}$ and is wavelength dependent.

\section{CONCLUSION}

In this paper, we have experimentally investigated the temperature induced dispersion coefficient for SMF-28 as a function of different wavelengths. Our results show good agreement with the value found in the literature for a narrow spectral spacing between wavelengths. In contrast, if using a wider spectral region of wavelengths (namely from $1545 \mathrm{~nm}$ to $1551 \mathrm{~nm}$ ) we found the changing dispersion slope for $D_{\text {temp }}$ This clearly indicates $D_{\text {temp }}$ wavelength dependency, i.e. $D_{\text {temp }}=D\left(\lambda_{n}\right)_{\text {temp. }}$. Therefore, applying Eq. (3) for finding the temporal width of the recovered $2 \mathrm{D}$ WH/TS IOCDMA auto-correlation function by the receiver when codes are based on multi-wavelength picosecond code carriers from a wider then a few nanometers spectral region requires to take into account the $D_{\text {temp }}$ wavelength dependency.

\section{REFERENCES}

[1] G. P. Agrawal, Nonlinear Fiber Optics. New York: Academic, 1989

[2] E.K.H. NG, G.E. Weichenberg, and E. H. Sargent, "Dispersion in Multiwavelength Optical Code-Division Multiple-Access Systems: Impact and Remedies," IEEE Trans Commun., vol. 50, no. 11, pp. 1811-1816, Nov. 2002.

[3] Md. S. Ahmed, M. I. Glesk, "Mitigation of Temperature Induced Dispersion in Optical Fiber on OCDMA Auto-correlation," IEEE Photon. Technol. Lett., vol. 29, no. 22, pp. 1979-1982, Nov. 2017).

[4] N. T. Dang, A.T. Pham, and Z. Cheng, "Impact of GVD on the Performance of 2-D WH/TS OCDMA System Using Heterodyne Detection Receiver," IEEE Trans. Fundamentals, vol. E92-A, no. 4, pp. 1182-1191, Apr. 2009.

[5] T. Kato, Y. Koyano, and M. Nishimura, "Temperature dependence of chromatic dispersion in various types of optical fiber," Optics Let., vol. 25, no. 16, pp. 1156-1158, Aug. 2000.

[6] T. B. Osadola, S. K. Idris, I. Glesk, I. Glesk, and W. C. Kwong, "Effect of Variations in Environmental Temperature on 2D-WH/TS OCDMA Code Performance," J. Opt. Commun. Netw., vol. 5, no. 1, pp. 68-73, Jan. 2013. 
[7] M. J. Hamp, J. Wright, M. Hubbard, and B. Brimacombe, "Investigation into the Temperature Dependence of Chromatic Dispersion in Optical Fiber," IEEE Photon. Technol. Lett., vol. 14, no. 11, pp. 1524-1526, Nov. 2002

[8] W. H. Hatton and M. Nishimura, "Temperature Dependence of Chromatic dispersion in Single Mode Fibers," J. Lightwave Technol., vol. LT-4, no. 10, pp. 1552-1555, Oct. 1986.

[9] P. S. Andre' and A. N. Pinto, "Chromatic dispersion fluctuations in optical fibers due to temperature and its effects in high-speed optical communication systems," Opt. Commun., vol. 246, no. 4-6, pp. 303 311, Feb. 2005
[10] M. S. Ahmed, M. Abuhelala, and I. Glesk, "Management of OCDMA auto-correlation function distorted by dispersion effects," in 19th International Conference on Transparent Optical Networks (ICTON 2017), Girona, Spain, 2-6 July 2017. DOI: 10.1109/ICTON.2017.8024789.

[11] K. S. Kim and M. E. Lines, "Temperature dependence of chromatic dispersion in dispersion-shifted fibers: Experiment and analysis," $J$. Appl. Phys., vol. 73, no. 5, pp. 2069-2074, March 1993.

[12] G. Ghosh, M. Endo, and T. Iwasaki, "Temperature-Dependent Sellmeier Coefficients and Chromatic Dispersions for Some Optical Fiber Glasses," J. Lightwave Technol., vol. 12, no. 8, pp.1338-1342, Aug. 1994. 